\title{
ON THE ORDER OF QUASIREGULAR MAPPINGS
}

\author{
S. RICKMAN and M. VUORINEN
}

\section{Introduction}

A continuous mapping $f: G \rightarrow R^{n}$ of a domain $G$ in the Euclidean $n$-space $R^{n}$, $n \geqq 2$, is called quasiregular (qr) if $f$ belongs to the local Sobolev space $W_{n, \text { loc }}^{1}(G)$ and for some $K, 1 \leqq K<\infty$,

$$
\left|f^{\prime}(x)\right|^{n} \leqq K J_{f}(x) \quad \text { a.e. }
$$

holds. Here $\left|f^{\prime}(x)\right|$ is the operator norm of the formal derivative $f^{\prime}(x)$ and $J_{f}(x)$ is the Jacobian determinant at $x$. Quasiregular mappings constitute a class of functions which is a natural generalization of the class of analytic functions in the complex plane. Quasimeromorphic (qm) mappings are maps into $\bar{R}^{n}=R^{n} \cup\{\infty\}$ and correspond in this theory to classical meromorphic functions. For some basic parts of the theory of qr and qm mappings the reader is referred to [5], [6], and [18].

For a nonconstant qm mapping $f: R^{n} \rightarrow \bar{R}^{n}$ we define the order $\mu_{f}$ and lower order $\lambda_{f}$ of $f$ by

$$
\begin{gathered}
\mu_{f}=\limsup _{r \rightarrow \infty} \frac{\log A(r)}{\log r}, \\
\lambda_{f}=\liminf _{r \rightarrow \infty} \frac{\log A(r)}{\log r} .
\end{gathered}
$$

Here $A(r)$ is the average of the counting function $n(r, y)$ with respect to the spherical $n$-measure in $\bar{R}^{n}, n(r, y)$ being the number of points of $f^{-1}(y)$ in the ball $\bar{B}(r)$, $B(r)=\left\{x \in R^{n}|| x \mid<r\right\}$, with multiplicity regarded.

If $G$ is a domain in $R^{n}$ and $f: G \rightarrow R^{n} \mathrm{qr}$, we write

$$
M(r)=\sup _{|x|=r}|f(x)| .
$$

For a nonconstant qr map $f: R^{n} \rightarrow R^{n}$ the order of $f$ can be defined by means of $M(r)$ as well, namely,

$$
\mu_{f}=\limsup _{r \rightarrow \infty}(n-1) \frac{\log \log M(r)}{\log r}
$$

holds and similarly for the lower order. This will be proved in Section 2 . 
In Theorem 3.3 we shall give a counterpart of the Phragmén-Lindelöf theorem for qr mappings. In a special case it says that if $f: R_{+}^{n} \rightarrow R^{n}$ is a nonconstant qr mapping of the half space $R_{+}^{n}=\left\{x \in R^{i n} \mid x_{n}>0\right\}$ with

$$
\limsup _{x \rightarrow y}|f(x)| \leqq 1
$$

for all $y \in \partial R_{+}^{n} \backslash\{\infty\}$, then either $|f|<1$ or

$$
\liminf _{r \rightarrow \infty} \frac{\log \log M(r)}{\log r} \geqq c(n, K)>0
$$

where $K$ is the number in (1.1). Another result is the following. Let $f: R^{n} \rightarrow R^{n}$ be nonconstant qr. If the lower order $\lambda_{f}<c(n, K) /(n-1)$, then $\lim \sup _{r \rightarrow \infty} m(r)=\infty$ where

$$
m(r)=\inf _{|x|=\boldsymbol{r}}|f(x)| .
$$

This is included in Theorem 3.5 and it is related to a result of Wiman [16, p. 121].

We have formulated our Phragmén-Lindelöf theorem for a qr mapping of an unbounded domain $G \subset R^{n}$ such that $R^{n} \backslash G$ is thick enough at $\infty$. A sufficient condition for the thickness will be given in terms of the $n$-capacity. By modifying a construction of an entire analytic function due to Toppila [15] we shall show that the thickness condition is in a sense best possible when $n=2$.

The proofs in Section 3 are based on a two-constant theorem for qr mappings from [11], which in turn is based on estimates on solutions of quasilinear partial differential equations due to Maz'ja [7]. Results related to those in Section 3 have been proved also in [1], [3], [8] and by K. Astala and S. Granlund.

\section{Equivalent definitions for the order}

Let $f: R^{n} \rightarrow \bar{R}^{n}$ be a nonconstant and $K$-quasimeromorphic mapping [6]. The counting function $n(r, y)$ is defined for $r>0, y \in \bar{R}^{n}$, by

$$
n(r, y)=\sum_{x \in f^{-1}(y) \cap \bar{B}_{(r)}(x, f)} i(x)
$$

where $i(x, f)$ is the local topological index; see [5, p. 6]. For $r, t>0$ we let $v(r, t)=$ $v_{f}(r, t)$ be the average of $n(r, y)$ over the sphere $S(t)=\partial B(t)$, i.e.

$$
v(r, t)=\frac{1}{\omega_{n-1}} \int_{S} n(r, t y) d \mathscr{H}^{n-1}(y)
$$

where $\mathscr{H}^{n-1}$ is the normalized $(n-1)$-dimensional Hausdorff measure, $S$ the unit sphere, and $\omega_{n-1}=\mathscr{H}^{n-1}(S)$. The following lemma is in a slightly weaker form in $[10,4.1]$. The present form is due to M. Pesonen and the proof can be found in [9] and [13]. 
2.1. Lemma. For $r, s, t>0$ and $\theta>1$

$$
v(\theta r, t) \geqq v(r, s)-\frac{K|\log (t / s)|^{n-1}}{(\log \theta)^{n-1}} .
$$

From 2.1 we get by integration (see $[10,4.8]$ )

$$
v(r / \theta, t)-\frac{K \tau}{(\log \theta)^{n-1}} \leqq A(r) \leqq v(\theta r, t)+\frac{K \tau}{(\log \theta)^{n-1}}, \quad r, t>0, \quad \theta>1,
$$

where $\tau>0$ depends only on $n$ and $t$. From (2.2) one gets the following lemma (see [10, 4.19] and [12, 2.4]).

2.3. Lemma. There exists a set $E \subset[1, \infty[$ of finite logarithmic measure, i.e.,

$$
\int_{E} \frac{d r}{r}<\infty
$$

such that

for all $t>0$.

$$
\lim _{E \nsubseteq r \rightarrow \infty} \frac{v(r, t)}{A(r)}=1
$$

Assume now that $\infty \notin f R^{n}$. For given $r>0$ and $\theta>1$ set $t>M(\theta r)$ and $s=1$ in 2.1. This gives

and hence

$$
0 \geqq v(r, 1)-\frac{K|\log t|^{n-1}}{(\log \theta)^{n-1}}
$$

$$
|\log M(\theta r)|^{n-1} \geqq \frac{(\log \theta)^{n-1}}{K} v(r, 1) .
$$

Let us look at the case where there exists a $\operatorname{limit}_{x \rightarrow \infty} f(x)$. Then $f$ extends to a qm $\operatorname{map} \tilde{f}: \bar{R}^{n} \rightarrow \bar{R}^{n}, \tilde{f}(\infty)=\infty$, and this case corresponds to a rational function in the classical theory. For any $t>0$ there exists $r_{0}(t)>0$ such that $v(r, t)$ is for $r \geqq r_{0}(t)$ the topological degree of $\tilde{f}$ which is $i(\infty, \tilde{f})$. By ideas from $[4,6.1]$ we can deduce that (for details, see [14, 5.4])

$$
K^{-1} v(r, 1)+o(1) \leqq \frac{|\log M(r)|^{n-1}}{(\log r)^{n-1}} \leqq K v(r, 1)+o(1) \quad(r \rightarrow \infty) .
$$

We will need in the general case also an inequality in the direction opposite to (2.4). We will give that in the following form where the factor $(\log r)^{n-1}$ appears like in (2.5).

2.6. Lemma. There exist $\theta_{1}=\theta_{1}(n, K)>1$ and $c_{1}=c_{1}(n, K)>0$ such that for some $r_{0}>1$

$$
v\left(\theta_{1} r, 1\right) \geqq c_{1} \frac{(\log M(r))^{n-1}}{(\log r)^{n-1}} \text { if } r \geqq r_{0} .
$$


Proof. We may assume that $\infty$ is an essential singularity. We will combine ideas from $[10,5.4]$ and $[11,3.2]$. Let $r>1$ and let $\Gamma$ be the family of paths in $B(2 r)$ which join $\bar{B}(1)$ and $f^{-1}\left(R^{n} \backslash B(M)\right)$ where $M=M(r)$. We may assume $M>1$. Then, by an $n$-dimensional version of [2, Theorem 4],

$$
M(\Gamma) \geqq \frac{d_{n}}{(\log r)^{n-1}}, \quad r \geqq r_{1},
$$

for some $r_{1}>1$ where $d_{n}>0$ is a constant which depends only on $n$. We first assume $M(1) \leqq 1$. Set

$$
\begin{aligned}
& \varrho(y)=\frac{3}{(\log M)|y|} \text { if } 1<|y|<M^{1 / 3}, \\
& \varrho(y)=0 \quad \text { elsewhere. }
\end{aligned}
$$

Then $\varrho$ is admissible for the family $f \Gamma$ and from the proof of $[5,3.2]$ we get that

$$
M(\Gamma) \leqq K \int_{R^{n}} n(2 r, y) \varrho(y)^{n} d m(y)=\frac{3^{n} K}{(\log M)^{n}} \int_{1}^{M^{1 / 3}} \frac{1}{\tau}\left(\int_{S} n(2 r, \tau z) d \mathscr{H}^{n-1}(z)\right) d \tau .
$$

Let $r \geqq r_{1}$. The inequalities (2.7) and (2.8) imply that there exists $y, 1 \leqq|y| \leqq M^{1 / 3}$, such that

$$
n(2 r, y) \geqq c_{1} \frac{(\log M)^{n-1}}{(\log r)^{n-1}},
$$

where $c_{1}=c_{1}(n, K)>0$. We separate two cases:

Case 1. For each $x \in f^{-1}(y) \cap \bar{B}(2 r)$ the $x$-component of $f^{-1} B\left(M^{2 / 3}\right)$ is contained in $B(4 r)$.

Let $x \in f^{-1}(y) \cap \bar{B}(2 r)$ and let $D$ be the $x$-component of $f^{-1} B\left(M^{2 / 3}\right)$. Then $D$ is a normal domain $([5,2.5])$ and

$$
\sum_{z \in f^{-1}(\zeta) \cap D} i(z, f)=\sum_{x \in f^{-1}(y) \cap D} i(x, f)
$$

for all $\zeta \in S$. By summing over all such components $D$ we obtain for all $\zeta \in S$

$$
n(4 r, \zeta) \geqq \sum_{D} \sum_{z \in f^{-1(\zeta) \cap D}} i(z, f)=\sum_{D} \sum_{x \in f^{-1}(y) \cap D} i(x, f) \geqq n(2 r, y) .
$$

Case 2. There exists $x \in f^{-1}(y) \cap \bar{B}(2 r)$ such that the $x$-component of $f^{-1} B\left(M^{2 / 3}\right)$ intersects $R^{n} \backslash B(4 r)$.

Let $\Gamma_{1}$ be the family of paths in $B(4 r)$ which join $f^{-1} B\left(M^{2 / 3}\right)$ and $f^{-1}\left(R^{n} \backslash B(M)\right)$. Then

$$
M\left(\Gamma_{1}\right) \geqq b=b(n)>0,
$$

and in place of (2.8) we now obtain

$$
M\left(\Gamma_{1}\right) \leqq \frac{3^{n} K \omega_{n-1}}{(\log M)^{n}} \int_{M^{2 / 3}}^{M} \frac{v(4 r, \tau)}{\tau} d \tau .
$$


These imply that there exists $t_{1} \in\left[M^{2 / 3}, M\right]$ such that

$$
v\left(4 r, t_{1}\right) \geqq \frac{b(\log M)^{n-1}}{3^{n-1} K \omega_{n-1}} .
$$

Let $2 C=b /\left(3^{n-1} K \omega_{n-1}\right)$. For $\theta_{1}>4$ Lemma 2.1 implies

$$
v\left(\theta_{1} r, 1\right) \geqq\left(2 C-\frac{K}{\left(\log \left(\theta_{1} / 4\right)\right)^{n}-1}\right)(\log M)^{n-1} .
$$

We choose $\theta_{1}>4$ so that $K\left(\log \left(\theta_{1} / 4\right)\right)^{1-n}=C$. The lemma follows then from (2.9), (2.10), and (2.11).

Let then $M_{1}=M(1)>1$. Let $g$ be the map $y \mapsto y / M_{1}$ and apply the above to the map $h=g \circ f$. If the constants for $h$ are $\theta_{1}$ and $c_{1}$, then

$$
v\left(\theta_{1} r, M_{1}\right)=v_{h}\left(\theta_{1} r, 1\right) \geqq c_{1} \frac{\left(\log \left(M / M_{1}\right)\right)^{n-1}}{(\log r)^{n-1}}, \quad r \geqq r_{1} .
$$

Since by 2.1

$$
v\left(2 \theta_{1} r, 1\right) \geqq v\left(\theta_{1} r, M_{1}\right)-\frac{K\left(\log M_{1}\right)^{n-1}}{(\log 2)^{n-1}}
$$

and since $v\left(\theta_{1} r, M_{1}\right) \rightarrow \infty$ as $r \rightarrow \infty \quad[10$, p. 455], we obtain

$$
v\left(2 \theta_{1} r, 1\right) \geqq \frac{c_{1}}{2} \frac{(\log M)^{n-1}}{(\log r)^{n-1}}, \quad r \geqq r_{0},
$$

for some $r_{0} \geqq r_{1}$. The lemma is proved.

After these preparations we are now ready to express the order and lower order in terms of the maximum norm function $M(r)$ as follows.

2.12. Theorem. Let $f: R^{n} \rightarrow R^{n}$ be a nonconstant qr mapping. Then

$$
\begin{aligned}
& \mu_{f}=\limsup _{r \rightarrow \infty}(n-1) \frac{\log \log M(r)}{\log r}, \\
& \lambda_{f}=\liminf _{r \rightarrow \infty}(n-1) \frac{\log \log M(r)}{\log r} .
\end{aligned}
$$

Proof. We shall prove (2.13). For (2.14) the proof is similar. Let the right hand side of (2.13) be $a$ and let $r_{i} \rightarrow \infty$ be a sequence of positive numbers such that $(n-1) \log \log M\left(r_{i}\right) / \log r_{i} \rightarrow a$. Let $\varepsilon>0$. By Lemma 2.6 there exists $\varrho>2$ such that

$$
a-\varepsilon \leqq \frac{\log v\left(\theta_{1} r_{i}, 1\right)}{\log r_{i}}, \quad r_{i} \geqq \varrho
$$


Let $E$ be the set given by Lemma 2.3. For any given $k>\varrho$ there exists by $2.3 r_{i} \geqq k$ and $s \in\left[\theta_{1} r_{i}, 2 \theta_{1} r_{i}\right] \backslash E$ such that $v(s, 1) \leqq A(s)(1+\varepsilon)$. Then

$$
a-\varepsilon \leqq \frac{\log v(s, 1)}{\log r_{i}} \leqq \frac{\log A(s)+\varepsilon}{\log s-\log \left(2 \theta_{1}\right)} .
$$

This gives $a \leqq \mu_{f}$. Similarly we get $a \geqq \mu_{f}$ by using (2.4) in place of 2.6. The theorem is proved.

\section{Phragmén-Lindelöf type theorems}

Let $(A, C)$ be a condenser in $R^{n}$, i.e. $A \subset R^{n}$ is open and $C \subset A$ compact. The capacity or $n$-capacity of $(A, C)$ is defined by

$$
\operatorname{cap}(A, C)=\inf _{u} \int_{A}|\nabla u|^{n} d m
$$

where $u$ runs through the set of functions in $C_{0}^{\infty}(A)$ with $u(x) \geqq 1$ for $x \in C$.

The following lemma is a two-constant theorem [11, 4.22]. Its proof is based on estimates from [7].

3.1. Lemma. Let $f: G \rightarrow R^{n}$ be a nonconstant $K$-qr mapping, $0<m<M$, and let $U=B(z, r)$ and

$$
\begin{gathered}
|f(x)| \leqq M \text { for } x \in G \cap U, \\
\limsup _{x \rightarrow y}|f(x)| \leqq m \text { for } y \in \partial G \cap \bar{U} .
\end{gathered}
$$

Then there are constants $\left.\lambda_{K} \in\right] 0,1 / 2[, C \in] 0,1[$ depending only on $n$ and $K$ such that if $0<\lambda \leqq \lambda_{K}$, then

$$
\log |f(x)| \leqq \beta_{0} \log m+\left(1-\beta_{0}\right) \log M
$$

for $x \in\left(G \backslash f^{-1}(0)\right) \cap B(z, \lambda r)$ where $\beta_{0} \in[0,1]$,

$$
\beta_{0}=C\left(\operatorname{cap}\left(B(z, r),\left(R^{n} \backslash G\right) \cap \bar{B}(z, \lambda r)\right)\right)^{1 /(n-1)} \log \frac{1}{i} .
$$

If $E \subset R^{n}$ is closed, $x \in R^{n}$, and if $r>0$, we set

$$
\begin{gathered}
\operatorname{cap}(E, r, x)=\operatorname{cap}(B(x, 2 r), E \cap \bar{B}(x, r)), \\
\operatorname{cap}(E, r)=\operatorname{cap}(E, r, 0) .
\end{gathered}
$$

By a result by Martio and Sarvas (cf. $[19,1.7]$ ) we have for $t>2 r$

$$
\operatorname{cap}(B(t), E \cap \bar{B}(r)) \geqq\left[\frac{\log (t / r)}{\log 2}\right]^{1-n} \operatorname{cap}(E, r) .
$$

In particular, we get a lower bound for the number $\beta_{0}$ in 3.1:

$$
\beta_{0} \geqq C(\log 2)\left(\operatorname{cap}\left(R^{n} \backslash G, \lambda r, z\right)\right)^{1 /(n-1)} .
$$

Our analogue of the Phragmén-Lindelöf theorem [16, p. 115] is the following. 
3.3. Theorem. Let $f: G \rightarrow R^{n}$ be a nonconstant $K$-qr mapping with

$$
\limsup _{x \rightarrow y}|f(x)| \leqq 1
$$

for all $y \in \partial G \backslash\{\infty\}$. If there exist numbers $\delta>0$ and $r_{0}>1$ such that cap $\left(R^{n} \backslash G, r\right) \geqq \delta$ for $r \geqq r_{0}$, then either $|f(x)|<1$ for $x \in G$ or

$$
\liminf _{r \rightarrow \infty} \frac{\log \log M(r)}{\log r} \geqq c(n, K, \delta)
$$

where $c(n, K, \delta)$ is a positive constant depending only on the dimension $n$, the maximal dilatation $K$, and the number $\delta$.

Proof. Suppose that $\left|f\left(x_{0}\right)\right| \geqq 1$ for some $x_{0} \in G$. Since a nonconstant qr mapping is open, there is $r_{1}>\max \left\{r_{0},\left|x_{0}\right|\right\}$ such that $M\left(r_{1}\right)>\left|f\left(x_{0}\right)\right|$. Let $\left.\lambda=\lambda_{K} \in\right] 0,1 / 2[$ and $C>0$ be the numbers in Lemma 3.1 and write $\beta=C(\log 2) \delta^{1 /(n-1)}$. Fix $r>r_{1} / \lambda$. Lemma 3.1 and (3.2) yield

$$
\begin{aligned}
& \log M(\lambda r) \leqq \beta \log 1+(1-\beta) \log M(r) \leqq(1-\beta) \log M(r) \\
& \log M\left(\lambda^{2} r\right) \leqq \beta \log 1+(1-\beta) \log M(\lambda r) \leqq(1-\beta)^{2} \log M(r) \\
& \quad \vdots \\
& \log M\left(\lambda^{k} r\right) \leqq(1-\beta)^{k} \log M(r) .
\end{aligned}
$$

Let $p=\max \left\{k \in N: \lambda^{k} r \geqq r_{1}\right\}$. Then $p \geqq\left(\log r_{1}-\log r\right) / \log \lambda-1$. In combination with the estimates above we get

and further

$$
\log M\left(r_{1}\right) \leqq(1-\beta)^{p} \log M(r)
$$

$$
\frac{\log \log M\left(r_{1}\right)}{\log r} \leqq\left(\frac{\log \left(r_{1} / r\right)}{\log \lambda}-1\right) \frac{\log (1-\beta)}{\log r}+\frac{\log \log M(r)}{\log r} .
$$

On letting $r \rightarrow \infty$ we get the desired bound with $c(n, K, \delta)=(\log (1-\beta)) / \log \lambda>0$.

3.4. Remarks. (1) To illustrate the meaning of the condition $\operatorname{cap}\left(R^{n} \backslash G, r\right) \geqq \delta$ in the theorem above we indicate a standard way of establishing adequate lower bounds. If $E \subset R^{n}$ is closed and $E_{r} \subset \bar{B}(r) \cap E$ is closed and connected, with $d\left(E_{r}\right) \geqq \tau r$, it follows from $[17,10.12]$ (cf. [19, 1.10]) that

$$
\operatorname{cap}(E, r) \geqq \operatorname{cap}\left(E_{r}, r\right) \geqq c_{n} \log \frac{4+\tau}{4-\tau}
$$

where $c_{n}>0$ is a constant depending only on $n$.

(2) Wallin [20, Theorem 4.4] has constructed a compact set $E$ in the unit ball of zero Hausdorff dimension with $\operatorname{cap}(E, 1)>0$. Applying dilations one can hence construct a closed set $F$ of zero Hausdorff dimension with cap $(F, r) \geqq \delta>0$ for $r \geqq 1([19,2.5])$.

For an entire qr mapping we get the following result by a similar proof. 
3.5. Theorem. Let $f: R^{n} \rightarrow R^{n}$ be a nonconstant $K$-qr mapping. If $E \subset R^{n}$ is a closed set such that there are numbers $\delta>0$ and $r_{0}>1$ with $\operatorname{cap}(E, r) \geqq \delta$ for $r \geqq r_{0}$, and

$$
\sup _{x \in E}|f(x)|<\infty
$$

then the lower order $\lambda_{f} \geqq d(n, K, \delta)$ where $d(n, K, \delta)>0$ is a constant depending only on $n, K$, and $\delta$. In particular, if $\lambda_{f}<d(n, K, \delta)$, then

where

$$
\limsup _{r \rightarrow \infty} m(r)=\infty
$$

$$
m(r)=\inf _{|x|=\boldsymbol{r}}|f(x)|
$$

Wiman [16, p. 121] has shown that an entire analytic function of order less than $1 / 2$ satisfies $\limsup _{r \rightarrow \infty} m(r)=\infty$. It is not known to the authors if results like Theorem 3.5 have been previously proved for analytic functions. Note that, in view of Remark 3.4 (2), the set $E$ in 3.5 can be rather small.

K. Astala and S. Granlund have obtained related results in forms where $\lambda_{f}$ has a lower bound $d$ which depends also on $\gamma=\limsup _{r \rightarrow \infty} \delta(r), \delta(r)=$ $\mathscr{H}^{n-1}(S(r) \cap G) /\left(\omega_{n-1} r^{n-1}\right)$ and $d / \infty$ when $\gamma \backslash 0$. Here $G=R^{n} \backslash E$. The results in [1] and [8] are also in this direction.

Theorem 3.5 implies immediately that $\lambda_{f} \geqq d(n, K)>0$ if $f: R^{n} \rightarrow R^{n}$ is nonconstant and has an asymptotic value in $R^{n}$. It is an open question whether the lower bound tends to $\infty$ when the number of asymptotic values tends to $\infty$.

\section{An example}

In this final section we shall give an example which shows that Theorem 3.5 is best possible in the sense described in Example 4.8 below. The example is obtained with minor modifications from a construction due to Toppila [15] as we shall now show.

For what follows fix an increasing function $h:] 0, \infty[\rightarrow] 0, \infty\left[\right.$ with $\lim _{t \rightarrow \infty} h(t)=\infty$. Consider the entire function

$$
f(z)=\prod_{j=1}^{\infty}\left(1-z / a_{j}\right)^{m_{j}}
$$

where $a_{j}, m_{j}>0$ are appropriately chosen sequences, the numbers $m_{j}$ being integers. The choice is made as follows. Let $a_{1}=m_{1}=9>e^{2}$. Suppose that $k \geqq 2$ and the numbers $a_{1}, \ldots, a_{k-1}, m_{1}, \ldots, m_{k-1}$ have been chosen. Choose $a_{k}$ such that

$$
\sum_{j=1}^{k-1} m_{j}<h\left(a_{k}\right)
$$


and

$$
\left(1+\sum_{j=1}^{k-1} m_{j}\right)^{2} a_{k-1}<\frac{a_{k}}{\log ^{2} a_{k}}
$$

Now choose an integer $m_{k}$ such that

$$
\sqrt{m_{k}} \geqq\left(\sum_{j=1}^{k-1} m_{j}\right) \log a_{k}>\sqrt{m_{k}}-1 \text {. }
$$

Conditions (4.3) and (4.4) have been slightly modified from the corresponding conditions in [15, pp. 5-6] for the proof of the second part of the following lemma.

4.5. Lemma. Let $f$ be defined by the formulae (4.1)-(4.4). Then $f$ enjoys the following properties:

$$
\begin{gathered}
\log M(r)=O\left(h(r)^{4} \log ^{6} r\right) \quad(r \rightarrow \infty) . \\
f(z) \rightarrow 0 \text { as } z \rightarrow \infty \text { and } z \in \cup B_{k}, B_{k}=\bar{B}\left(a_{k}, a_{k} / 4\right) .
\end{gathered}
$$

Proof. The proof of (1) can be done, with obvious changes, in the same manner as in [15, pp. 6-7], where the reader can find the details. The proof of (2) is much shorter and based on ideas from [15]. We shall give here only the proof of (2).

To prove (2), we obtain for $z \in B_{k}$

$$
\begin{gathered}
\log |f(z)| \leqq \sum_{j=1}^{\infty} m_{j} \log \left|1-z / a_{j}\right| \leqq \\
\sum_{j=1}^{k-1} m_{j} \log \left(1+|z| / a_{j}\right)+m_{k} \log (1 / 4)+\sum_{j=k+1}^{\infty} m_{j}\left(5 a_{k} /\left(4 a_{j}\right)\right) .
\end{gathered}
$$

It follows from (4.3) and (4.4) that (cf. [15, p. 6])

and $a_{j}>400 a_{j-1}$. Hence

$$
m_{j} / a_{j}<\frac{1}{a_{j}}\left(1+\sum_{i=1}^{j-1} m_{i}\right)^{2} \log ^{2} a_{j}<\frac{1}{a_{j-1}}
$$

$$
\sum_{j=k+1}^{\infty} m_{j}\left(5 a_{k} /\left(4 a_{j}\right)\right)<2
$$

The estimates (4.6) and (4.7) together with $\log \left(1+|z| / a_{j}\right) \leqq \log (2|z|)$ yield

$$
\log |f(z)| \leqq\left(\sum_{j=1}^{k-1} m_{j}\right) \log \left(5 a_{k} / 2\right)+m_{k} \log (1 / 4)+2=m_{k}(\log (1 / 4)+\varepsilon(k))
$$

Here $\varepsilon(k) \rightarrow 0$ as $k \rightarrow \infty$ by (4.4). The proof is complete.

4.8. Example. Let $f: R^{2} \rightarrow R^{2}$ be a nonconstant entire analytic function of order zero with positive zeros $a_{1}<a_{2}<\ldots, \lim a_{j}=\infty$, such that if $B_{k}=\bar{B}\left(a_{k}, a_{k} / 4\right)$, 
then for some $k_{0} \geqq 1$,

$$
|f(x)|<1 \quad \text { for } \quad x \in \bigcup_{k \geqq k_{0}} B_{k} .
$$

Choosing $h(r)=\log (1+r)$ in Lemma 4.5 we see that such functions exist. Let $E=\bigcup_{k \geqq k_{0}} B_{k}$. By Remark 3.4 (1) there is $\tau>0$ such that cap $\left(E, a_{k}\right) \geqq \tau$ for $k \geqq k_{0}$. By (4.9)

$$
\limsup _{x \rightarrow y}|f(x)| \leqq 1
$$

for all $y \in E$. Since $\mu_{f}=0$, we see that the condition cap $(E, r) \geqq \delta>0$, for large $r>0$, in Theorem 3.5 cannot be replaced by the corresponding condition for a sequence of numbers $r_{k} \nearrow \infty$ when $n=2$. On the other hand, it would be enough to assume $\operatorname{cap}\left(E, s_{k}\right) \geqq \delta>0$ for a sequence $\left(s_{k}\right)$ with, say, $s_{k+1}<2 s_{k}$ for all $k$.

\section{References}

[1] Botvinnik, V. A., and V. M. Mikljukov: A theorem of Phragmén-Lindelöf type for n-dimensional mappings with bounded distortion. - Sibirsk. Mat. Ž. 21, 1980, 232235, 240 (Russian).

[2] Gehring, F. W.: Symmetrization of rings in space. - Trans. Amer. Math. Soc. 101, 1961, $499-519$.

[3] Granlund, S., P. Lindevist, and O. Martio: F-harmonic measure in space. - To appear.

[4] Martio, O.: A capacity inequality for quasiregular mappings. - Ann. Acad. Sci. Fenn. Ser. A I Math. 474, 1970, 1-18.

[5] Martio, O., S. RickMan, and J. VäISÄL $\ddot{A}:$ Definitions for quasiregular mappings. - Ann. Acad. Sci. Fenn. Ser. A I Math. 448, 1969, 1-40.

[6] Martio, O., S. RickMAN, and J. VÄIs̈̈L $\ddot{A}:$ Distortion and singularities of quasiregular mappings. - Ann. Acad. Sci. Fenn. Ser. A I Math. 465, 1970, 1-13.

[7] MAZ'JA, V. G.: The continuity at a boundary point of the solutions of quasilinear elliptic equations. - Vestnik Leningrad. Univ. 25, no. 13, 1970, 42-55 (Russian).

[8] Mikluukov, V. M.: Asymptotic properties of subsolutions of quasilinear equations of elliptic type and mappings with bounded distortion. - Mat. Sb. (N.S.) 111 (153). 1980, no. 1, 42-66, 159 (Russian).

[9] Pesonen, M.: A path family approach to Ahlfors's value distribution theory. - Ann. Acad. Sci. Fenn. Ser. A I Math. Dissertationes 39, 1982, 1-32.

[10] Rickman, S.: On the value distribution of quasimeromorphic maps. - Ann. Acad. Sci. Fenn. Ser. A I Math. 2, 1976, 447-466.

[11] RickMAN, S.: On the number of omitted values of entire quasiregular mappings. - J. Analyse Math. 37, 1980, 100-117.

[12] Rickman, S.: A defect relation for quasimeromorphic mappings. - Ann. of Math. (2) 114, 1981, 165-191.

[13] Rickman, S.: Value distribution of quasiregular mappings. - Proceedings of the Nordic Summer School in Mathematics, Joensuu, 1981. Lecture Notes in Mathematics, Springer-Verlag, Berlin-Heidelberg-New York (to appear).

[14] SRebro, U.: Quasiregular mappings. - Proceedings of Seminars held at Maryland University 1973/1974. Lecture Notes in Mathematics 505, Springer-Verlag, Berlin-Heidelberg -New York, 1976, 148-163. 
[15] Toppila, S.: Some remarks on exceptional values at Julia lines. - Ann. Acad. Sci. Fenn. Ser. A I Math. 456, 1970, 1-20.

[16] Tsusi, M.: Potential theory in modern function theory. - Chelsea Publishing Co, New York, 1975.

[17] VÄIs ÄL $\ddot{A}$, J.: Lectures on $n$-dimensional quasiconformal mappings. - Lecture Notes in Mathematics 229, Springer-Verlag, Berlin-Heidelberg-New York, 1971.

[18] VÄIS̈̈L $\ddot{A}$, J. : A survey of quasiregular maps in $R^{n}$. - Proceedings of the International Congress of Mathematicians, Helsinki, Finland, August 15-23, 1978, Helsinki, 1980, 685-691.

19] VUORINEN, M.: On the existence of angular limits of $n$-dimensional quasiconformal mappings. - Ark. Mat. 18, 1980, 157-180.

[20] Wallin, H.: Metrical characterizations of conformal capacity zero. - J. Math. Anal. Appl. $58,1977,298-311$.

University of Helsinki

Department of Mathematics

SF-00100 Helsinki 10

Finland

Received 14 January 1982 